\title{
Research on Effects of Obstacles on Heat Transfer and Fluid Flow in Backward Facing Step Flow
}

\author{
S.Ramanathan, S.Prabhu, R. S. Mohankumar, T.Suresh
}

\begin{abstract}
An extensive research has been done on heat transfer and pressure drop characteristics in micro-channel using liquid water. A baffle has been introduced downstream the sudden expansion zone to enhance the rate of heat transfer. The height and the location of the baffle were varied for the Reynolds number range 50 $\leq \mathrm{Re} \leq 200$, which is a laminar flow. Twodimensional flow domain with non-staggered grid arrangement was taken and the two-dimensional mass, momentum and energy equation was solved using finite volume method in ANSYS 16.2. This study reports that the presence of baffle in the microchannel increased the rate of heat transfer. The skin friction coefficient has been calculated and the parameters influencing the heat transfer augmentation have been optimized.
\end{abstract}

Keywords - Heat transfer, Micro channels, Laminar flow, Skin friction coefficient

\section{INTRODUCTION}

Many researches have been conducted in the microchannels with backward facing step for predicting the flow characteristics and to augment the heat transfer. The recirculation formation and reattachment in the flows are crucial in escalating the heat transfer and pressure drop characteristics in the micro channels with step. This recirculation branches into primary, secondary and tertiary during the flows. This primary recirculation with the contribution of secondary and tertiary recirculation created through the backward facing step influence fluid flow and boosts heat transfer. The channels with increase in step height increases the skin friction coefficient and decreases the pressure drop in laminar flow regimes[1]. Hamid E. Ahmed et al., used longitudinal Vortex generators (VGs) as turbulators in the microchannels with backward facing step for enhancing the heat transfer and pressure drop characteristics. Also, the attack angle has been varied for different VGs with and without winglet pairs and enhanced the heat transfer with rectangular VGs for attack angle of 60 degree with the little increase in pressure drop[2].The longitudinal vortex generators as rectangular winglet pair is introduced in the rectangular channels and the effects of skin friction coefficient and heat transfer characteristics are enhanced for laminar flow regions[3]. S. Chakrabarti et al., attempted the microchannels with fence for various subtended angles and concluded that the fence increases or

Revised Manuscript Received on July 18, 2019.

S.Ramanathan, Department of Mechanical Engineering, Kumaraguru College of Technology, Coimbatore -641049, India.

S.Prabhu, Department of Mechanical Engineering, Kumaraguru College of Technology, Coimbatore -641049, India.

R. S. Mohankumar, Department of Mechanical Engineering, Kumaraguru College of Technology, Coimbatore -641049, India.

T.Suresh, Department of Mechanical Engineering, Kamaraj College of Engineering and Technology, Madurai- 625 701, India. decreases the performance of micro channels for lower Re number range[4]. Hence, usage of fence may sometimes enhance the heat transfer in the sudden expansion zones. The step angle in the microchannels has also been varied to predict the performance of sudden expansion zone for the higher Re range (say $\operatorname{Re}>5000$ ) with different expansion ratios. The turbulence effects has been astutely predicted with Large Eddie Simulation approach which shows that the reattachment length, flow separation and flow structure shows very good agreement besides the step[5]. M. Thiruvengadam et al, analysed the channels with the effect of buoyance forces and duct aspect ratio for the laminar flow regimes and heat transfer characteristics with mixed convection and plane symmetric sudden expansion zones. The variation in the buoyancy force increases the recirculation zone and decreases the reattachment length when move towards the sudden expansion zone in turn increases the heat transfer[6]. However, the flow characteristics and heat transfer effects are predicted for the critical laminar flow regime $\mathrm{Re}=114$ for the different baffle heights. The study shows that, increase in the baffle height with position nearer to the step enhances heat transfer and skin friction coefficient[7]. The channels with backward facing step for various step angles have been analysed for different incompressible turbulent flows. Finite volume method has been used for predicting the simulation and the references as per the simulation is applied to the experimental data. This provides good agreement with lower inclination angles and for the higher inclination angles the recirculation zone is difficult to predict[8]. Moreover, the large eddie simulation approach has been used by Kazuaki sugawara et al., for the turbulent flows to predict the heat transfer in the symmetric expansion plane channel. Here, the flow characteristics was analysed for only one $\mathrm{Re}=15000$ and it provides good agreement with the experimental values [9].

Many researches have been conducted in the microchannels with backward facing step for augmenting the heat transfer for the different laminar and turbulent flows. This has been done with introducing fences, jet impingements, baffles, vortex generators and by changing the angle of inclination of the step. The variation of baffle heights with the different positions away from the step in the laminar flow has not been explored so far. In this study, an attempt has been made to enhance the parameters

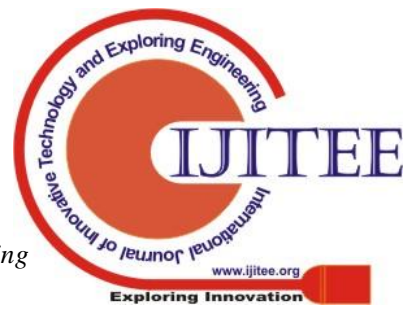




\section{RESEARCH ON EFFECTS OF OBSTACLES ON HEAT TRANSFER AND FLUID FLOW IN BACKWARD FACING STEP FLOW}

influencing the heat transfer by placing the baffle of different heights with the various positions away from the backward facing step. The simulation has been performed in the different laminar flow regimes to predict effects of baffle on the skin friction coefficient and $\mathrm{Nu}$ which augments the heat transfer.

\section{NUMERICAL METHOD AND GRID INDEPENDENT STUDY}

The geometry was created in ANSYS Workbench Design Modeler and the same was meshed in ICEM CFD as shown in Fig. 1. A fine and uniform quadrilateral mesh was adopted in a computational domain so that the flow physics are captured precisely. Continuity, momentum and energy equations were solved till residuals became less than 10-6. FLUENT was used to solve the boundary conditions and the governing equations. Nusselt number was computed for different grid systems. The numerical solutions were examined to ascertain that they are independent of grid number as given in the Table 1 . The relative variation of the Nusselt number for 1620000 and 2016224 grid numbers was less than 0.08 and grid number of 1620000 was adopted for the domain to carry out CFD analysis in FLUENT.

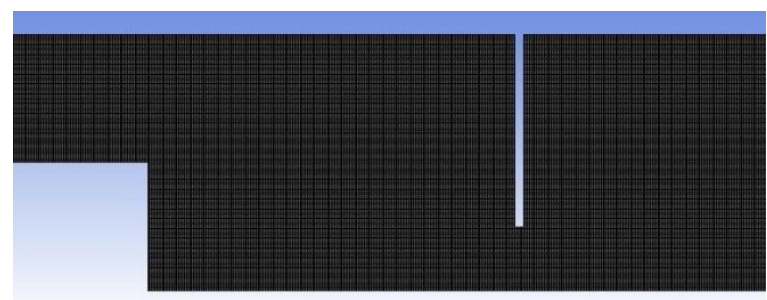

Fig. 1. Mesh pattern

TABLE 1

\begin{tabular}{|c|c|}
\hline Elements & Nu \\
\hline 451984 & 64.25892 \\
\hline 1036800 & 64.32778 \\
\hline 1620000 & 64.39599 \\
\hline 2016224 & 64.44326 \\
\hline
\end{tabular}

\section{MODEL FORMULATION}

The computational domain of the micro channel is shown in Fig. 2.The length of the upstream and downstream channel of step was 240 and $1500 \mathrm{~mm}$ to ensure fully developed flow both at the inlet and outlet. The height of the baffle has been varied in steps of $5 \mathrm{~mm}$, from $10 \mathrm{~mm}$ to 30 $\mathrm{mm}$. The height of the inlet and step is $20 \mathrm{~mm}$. The location of the baffle has been varied with $20 \mathrm{~mm}$ incremental from 280 to $360 \mathrm{~mm}$ in downstream inlet. Fluid with $\operatorname{Pr}=0.7$ enters into the channel at $293 \mathrm{~K}$ with various uniform velocities corresponding to different $\mathrm{Re}$ in which the flow has been studied. The Re for the analysis are taken as 50, 100,150 and 200 after which the flow remains uniform. Also, uniform heat flux of $1 \mathrm{~kW}$ is supplied at bottom wall and remaining all other walls are modelled as adiabatic.

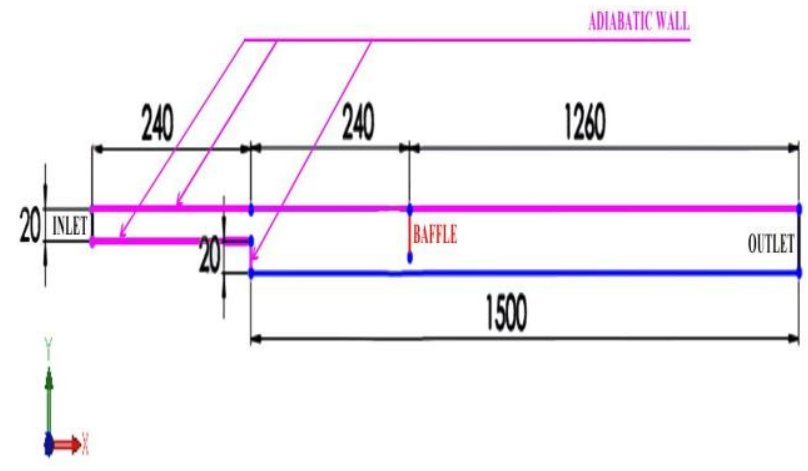

Fig. 2. Computational domain

\section{RESULTS AND DISCUSSION}

\section{a. Effect of baffle on the fluid flow}

As many studies indicates that, the presence of baffle in the micro channels increases the heat transfer but the height and location of baffle in the micro channel which contributes the heat transfer augmentation have to be explored. The prediction of the velocity contours for the fluid flows with different $\mathrm{Re}$ when the baffle is located at the regions $X=280$ to $X=360 \mathrm{~mm}$ from the step are shown in the fig. 3- 10. The simulation study shows a strong primary recirculation occurs in the step wall aside of the baffle and the clear jet like impingement occurs under the baffle. Also, the secondary recirculation near the baffle and the tertiary recirculation formed in the bottom wall is shown in the velocity contour profile figures $3-10$. In this fluid flow study, the height of the baffle has been varied from 10 to 30 $\mathrm{mm}$ with $5 \mathrm{~mm}$ incremental for the various $\mathrm{Re}$ and the simulation results shows that the maximum heat transfer occurs for the baffle with 30mm height. Moreover, in our previous study, it has been found that the primary recirculation gets weakened when the baffle is positioned beyond $560 \mathrm{~mm}$ in the micro channels.

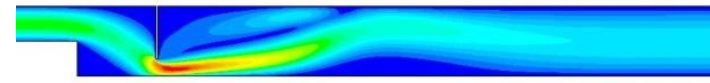

Fig. 3. Velocity contour of the fluid flow $\mathrm{Re}=\mathbf{5 0}$ with $30 \mathrm{~mm}$ baffle at $X=280 \mathrm{~mm}$

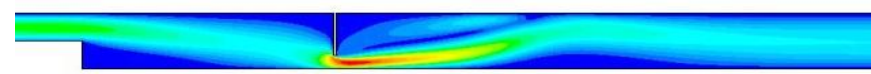

Fig. 4. Velocity contour of the fluid flow $R e=50$ with $30 \mathrm{~mm}$ baffle at $X=360 \mathrm{~mm}$

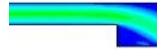

Fig. 5. Velocity contour of the fluid flow $\mathrm{Re}=100$ with $30 \mathrm{~mm}$ baffle at $X=280 \mathrm{~mm}$ 
Fig. 6. Velocity contour of the fluid flow $\mathrm{Re}=100$ with $30 \mathrm{~mm}$ baffle at $X=360 \mathrm{~mm}$

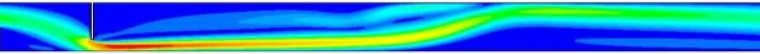

Fig. 7. Velocity contour of the fluid flow $\mathrm{Re}=\mathbf{1 5 0}$ with $30 \mathrm{~mm}$ baffle at $X=280 \mathrm{~mm}$

Fig. 8. Velocity contour of the fluid flow $\mathrm{Re}=\mathbf{1 5 0}$ with $30 \mathrm{~mm}$ baffle at $\mathrm{X}=360 \mathrm{~mm}$

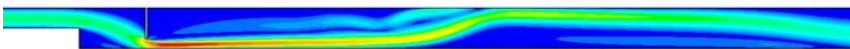

Fig. 9. Velocity contour of the fluid flow $\mathrm{Re}=\mathbf{2 0 0}$ with $30 \mathrm{~mm}$ baffle at $X=280 \mathrm{~mm}$

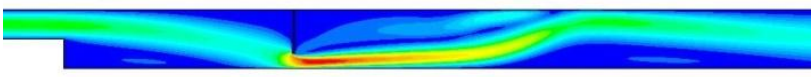

Fig. 10. Velocity contour of the fluid flow $R e=200$ with $30 \mathrm{~mm}$ baffle at $X=360 \mathrm{~mm}$

It has been found in the simulation study that, the skin friction coefficient gets astutely increased in the recirculation zone i.e., in the baffle position with the abrupt drop in pressure in the downstream region of the baffle. Variation of skin friction coefficient along the channel for various $R e$ with the $30 \mathrm{~mm}$ baffle height from the positions $\mathrm{X}=280$ to $360 \mathrm{~mm}$ is shown in the fig. 11-14. The figures show that,the skin friction coefficient gets constant throughout the channel after the baffle position.

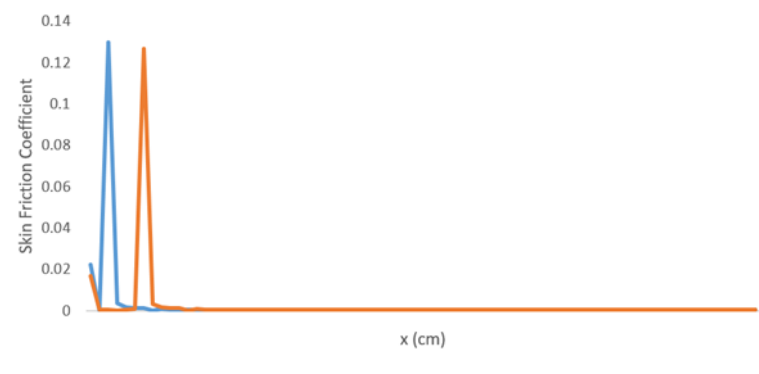

Fig. 11. Skin friction coefficient for the fluid flow $\mathbf{R e}=\mathbf{5 0}$ with $30 \mathrm{~mm}$ baffle height

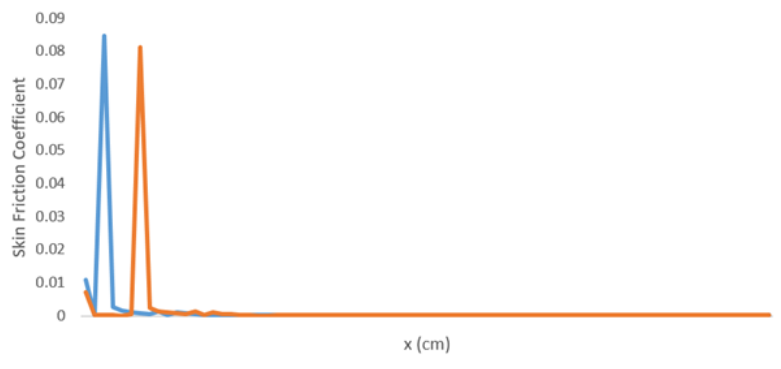

Fig. 12. Skin friction coefficient for the fluid flow $\mathbf{R e}=100$ with $30 \mathrm{~mm}$ baffle height

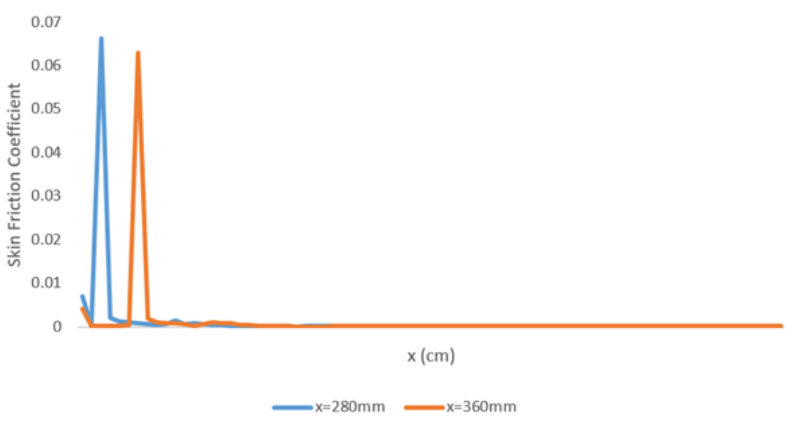

Fig. 13. Skin friction coefficient for the fluid flow $\mathbf{R e}=\mathbf{1 5 0}$ with $30 \mathrm{~mm}$ baffle height

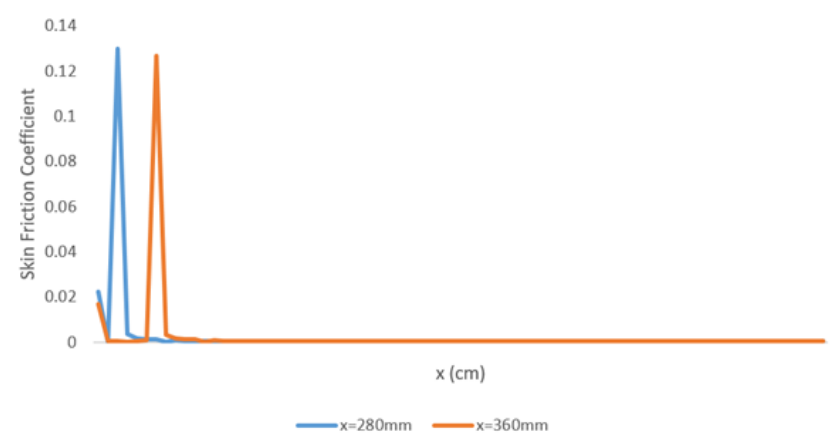

Fig. 14. Skin friction coefficient for the fluid flow $\mathbf{R e}=200$ with $30 \mathrm{~mm}$ baffle height

\section{b. Effect of baffle on heat transfer}

It is well known that the $\mathrm{Nu}$ is the important parameter in predicting the heat transfer. For the fluid flow different Re, the $\mathrm{Nu}$ variation along the length of the channel is depicted in the following fig. 15-18. The study shows that, $\mathrm{Nu}$ is higher for the flow $\mathrm{Re}=200$ when the baffle height is maximum. It indicates the heat transfer is very high for the above flow due to the presence of baffle with $30 \mathrm{~mm}$ height.This bound the conclusion; the heat transfer proportionately increases with the increase in baffle height for fluid flows with various Re.Also, this sharp increasing trend of $\mathrm{Nu}$ at the baffle positions with different baffle heights nearer to the step is found same for various flow regimes. At the same time, it is predicted that, the $\mathrm{Nu}$ decreases with the baffle positions away from the step is found common in the analysis for various fluid flows. So the baffle positioned in the top wall of the channel nearer to the

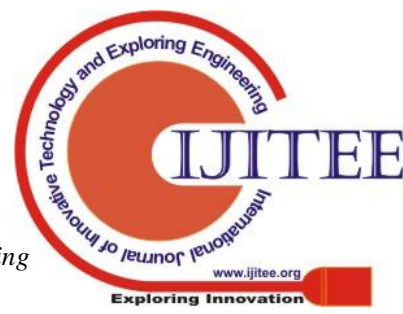




\section{RESEARCH ON EFFECTS OF OBSTACLES ON HEAT TRANSFER AND FLUID FLOW IN BACKWARD FACING STEP FLOW}

step directs the flow which escalates the heat transfer such that, the heat transfer rate increases due to the formation of primary recirculation till the fluid approaches the reattachment. After which, the heat transfer gets decreased and the same trend falls common for all the laminar flow regimes. This indicates that the baffle has no control on recirculation when it is away from the step but still it enhances heat transfer by forming eddies.

Formation of eddies is common in the baffle positions irrespective of its positions from the step. Eddies formed during the flow gets higher with the increase in the baffle height for the same position from the step. These eddies significantly contribute for increasing the heat transfer for the position of the bafflewith maximum height nearer to the step. When the baffle is away from the step, the recirculation gets out of bounds hence formation eddies enhances the heat transfer.

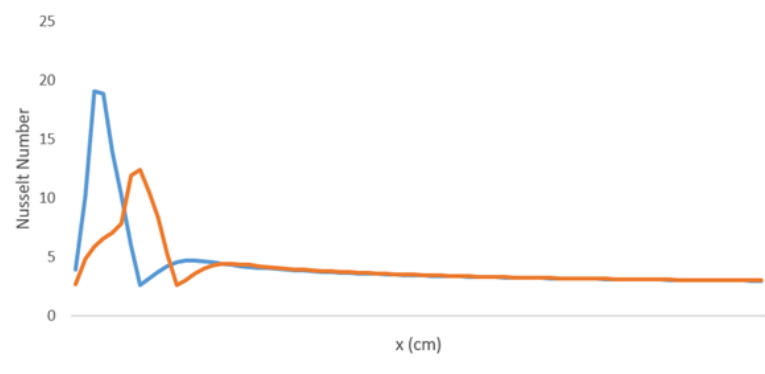

Fig. 15. Variation of $\mathrm{Nu}$ for the fluid flow $\mathrm{Re}=50$ with 30 mm baffle height

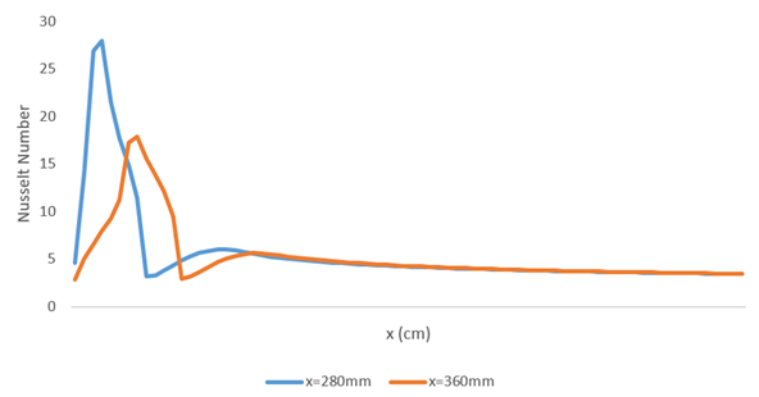

Fig. 16. Variation of $\mathrm{Nu}$ for the fluid flow $\mathrm{Re}=100$ with $30 \mathrm{~mm}$ baffle height

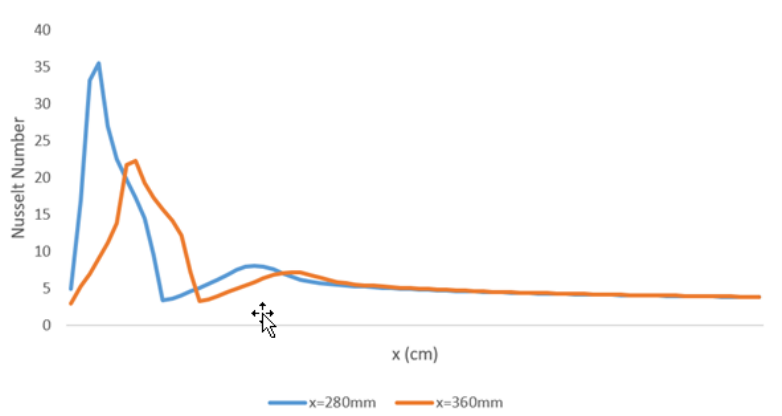

Fig. 17. Variation of $\mathrm{Nu}$ for the fluid flow $\mathrm{Re}=150$ with $30 \mathrm{~mm}$ baffle height

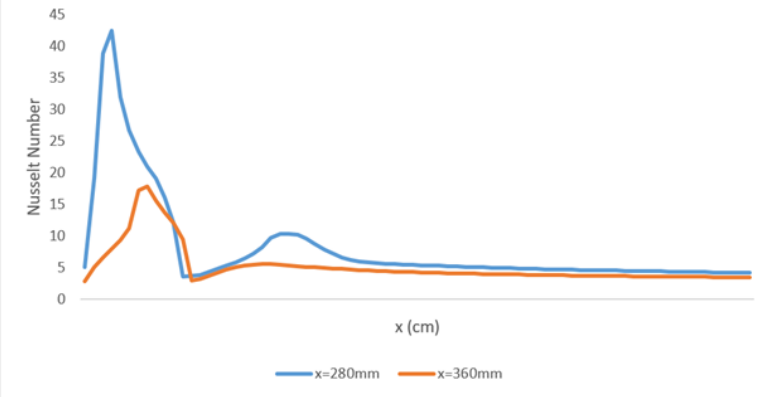

Fig. 18. Variation of $\mathrm{Nu}$ for the fluid flow $\mathrm{Re}=\mathbf{2 0 0}$ with $30 \mathrm{~mm}$ baffle height

\section{CONCLUSION}

The baffle in the micro channels with backward facing step increases the heat transfer due to the enhancement of primary recirculation with the contribution of larger eddies.

The heat transfer is maximum for the baffles nearer to the step, with increase in the baffle height. Also, increase in baffle heights increases heat transfer for all the baffle positions due to the formation of smaller eddies.

The heat transfer decreases for the baffle when it is positioned in increasing order from the step. This phenomenon is common for all the baffle heights.

$\begin{array}{ll}\text { Nomenclature } \\ \mathrm{Nu} & \text { Nusselt Number } \\ \mathrm{X} & \text { Cartesian coordinate }(\mathrm{m}) \\ \mathrm{H} & \text { Baffle height }(\mathrm{m}) \\ \mathrm{Re} & \text { Reynolds Number } \\ \mathrm{Pr} & \text { Prandtl Number }\end{array}$

\section{ACKNOWLEDGMENT}

Authors thank the management of Kumaraguru College of Technology, Coimbatore - India for providing facilities to carry out the research work.

\section{REFERENCES}

1. A. S. Kherbeet, H. A. Mohammed, K. M. Munisamy, and B. H. Salman, "The effect of step height of microscale backwardfacing step on mixed convection nanofluid flow and heat transfer characteristics," Int. J. Heat Mass Transf., vol. 68, pp. 554-566, 2014.

2. H. E. Ahmed, A. S. Kherbeet, M. I. Ahmed, and B. H. Salman, "Heat transfer enhancement of micro-scale backward-facing step channel by using turbulators," Int. J. Heat Mass Transf., vol. 126, pp. 963-973, 2018.

3. Q. Zhang and L. B. Wang, "Numerical study of heat transfer enhancement by rectangular winglet vortex generator pair in a channel," Adv. Mech. Eng., vol. 8, no. 5, pp. 1-11, 2016.

4. S. Chakrabrti, S. Rao, and D. K. Mandal, "Numerical Simulation of the Performance of a Sudden Expansion With Fence Viewed as a Diffuser in Low Reynolds Number Regime," J. Eng. Gas Turbines Power, vol. 132, no. 11, p. 114502, 2010.

5. H. H. Choi, V. T. Nguyen, and J. Nguyen, "Numerical Investigation of Backward Facing Step Flow over Various Step Angles," Procedia Eng., vol. 154, no. 1983, pp. 420-425, 2016. 
6. M. Thiruvengadam, B. F. Armaly, and J. A. Drallmeier, "Three dimensional mixed convection in plane symmetricsudden expansion: Symmetric flow regime," Int. J. Heat Mass Transf., vol. 52, no. 3-4, pp. 899-907, 2009.

7. R. Jayakumar, J. S. J, S. Prabhu, A. P. Arun, P. Karthi, and S. Ramanathan, "Numerical Heat Transfer Analysis in a MicroChannel with a Baffle" "International conference on Automotive systems, Agricultural Equipments and Manufacturing (ICAAM 2017) ICAAM 2017 - Conference Proceedings," no. Icaam, 2017.

8. P. Louda, J. Př́íhoda, K. Kozel, and P. Sváček, "Numerical simulation of flows over 2D and 3D backward-facing inclined steps," Int. J. Heat Fluid Flow, vol. 43, pp. 268-276, 2013.

9. K. Sugawara, H. Yoshikawa, and T. Ota, "LES of Turbulent Separated Flow and Heat Transfer in a Symmetric Expansion Plane Channel,” J. Fluids Eng., vol. 127, no. 5, p. 865, 2005. 